\title{
Algebraic Multigrid Solver on Clusters of CPUs and GPUs
}

\author{
Aurel Neic, Manfred Liebmann, and Gundolf Haase \\ Institute for Mathematics and Scientific Computing, University of Graz, Austria ${ }^{\star \star}$ \\ aurel.neic@uni-graz.at
}

\begin{abstract}
Solvers for elliptic partial differential equations are needed in a wide area of scientific applications. We will present a highly parallel CPU and GPU implementation of a conjugate gradient solver with an algebraic multigrid preconditioner in a package called Parallel Toolbox. The solvers operates on fully unstructured discretizations of the PDE. The algorithmic specialities are investigated with respect to many-core architectures and the code is applied to one current application. Benchmark results of computations on clusters of CPUs and GPUs will be presented. They will show that a linear equation system with 25 million unknowns can be solved in about 1 second.
\end{abstract}

Keywords: Algebraic multigrid, GPU computing, High performance computing

\section{Introduction}

As the finite-element approach for solving elliptic partial differential equations is very popular in many scientific applications, fast solvers for the resulting linear equation systems are of great interest. In this paper we want to present a parallel CPU and GPU implementation of a conjugate gradient solver using an algebraic multigrid preconditioner (AMG) called Parallel Toolbox. The basic ideas behind the parallelization concept as well as the differences between the CPU and the GPU implementation will be investigated.

Our code is designed for a fast AMG implementation on clusters of CPUs and GPUs for special problems as the application project described in \$2. In contrast, the more general applicable AMG-code BOOMER/HYPRE requires longer setup times and is not suited for GPUs but performs very well on large numbers of CPUs $[1,5]$. There exist codes for Multigrid on GPUs but all of them require structured grids or at least locally structured grids as in FEAST [10,3]. Therfore, our implementation is the first multigrid solver on GPUs for fully unstructured discretizations of the underlying problem elliptic partial differential equations.

\footnotetext{
$\star \star$ The final publication can be found at http://www.springer.com/computer/theoretical+computer+science/book/978-3-642-28144-0
} 


\section{The CARP project}

Let us first introduce the application project where our toolbox has been integrated. The Cardiac Arrhythmias Research Package is a project for the electrophysiological simulation of cardiac tissue which has been developed by Dr. Edward Vigmond and Dr. Gernot Plank [12]. Because of the great research activity in this area, CARP has a plug-in framework allowing new ionic models to be easily implemented.

By design CARP can run on shared computers as well as on distributed computers. Parallelization is performed within the shared memory model by implementing OpenMP directives and native numerical libraries. For distributed memory parallelization, extensive use of the PETSc parallel library as well as MPI function calls is made. In addition, efforts have been made to implement the Parallel Toolbox into CARP.

\subsection{Components}

The package consists of three main components: a parabolic solver, an ionic current component, and an elliptic solver. Each of these components has a set of APIs for connection to other components. The parabolic solver is responsible for determining the propagation of electrical activity by determining the change in transmembrane voltage from the extracellular electric field and the current state of the transmembrane voltage.

The elliptic solver unit determines extracellular potential from transmembrane voltage at each time step. The ionic model component is computed from a separate library which must be linked in at compile time.

\subsection{Mathematical model}

The most complete description of cardiac electricity is given by the bidomain equations (see [12]). The basic bidomain equations relate the intracellular potential $\phi_{i}$ to the extracellular potential $\phi_{e}$ through the transmembrane current density $I_{m}$ :

$$
\begin{aligned}
\nabla \cdot \bar{\sigma}_{i} \nabla \phi_{i} & =\beta I_{m} \\
\nabla \cdot \bar{\sigma}_{e} \nabla \phi_{e} & =-\beta I_{m}-I_{e} \\
I_{m} & =C_{m} \frac{\partial V_{m}}{\partial t}+I_{\text {ion }}-I_{\text {trans }}
\end{aligned}
$$

where $\bar{\sigma}_{i}$ and $\bar{\sigma}_{e}$ are respectively the intracellular and extracellular conductivity tensors, $\beta$ is the surface to volume ratio of the cardiac cells, $I_{\text {trans }}$ denotes the transmembrane current density stimulus as delivered by the intracellular electrode, $I_{e}$ is an extracellular current density stimulus, $C_{m}$ is the capacitance per unit area, $V_{m}$ is the transmembrane voltage which is defined as $\phi_{i}-\phi_{e}$, and $I_{i o n}$ refers to the current density flowing through the ionic channels. Eqn. 3 is a set of ordinary differential equations which can be solved independently for each node of the spacial discretization.

By adding Eqn. 1 and Eqn. 2 and using the definition of $V_{m}$, the equations can be cast in a slightly different form with $V_{m}$ and $\phi_{e}$ as independent variables.

$$
\begin{aligned}
\nabla \cdot\left(\bar{\sigma}_{i}+\bar{\sigma}_{e}\right) \nabla \phi_{e} & =-\nabla \cdot \bar{\sigma}_{i} \nabla V_{m}-I_{e} \\
\nabla \cdot \bar{\sigma}_{i} \nabla V_{m} & =-\nabla \cdot \bar{\sigma}_{i} \nabla \phi_{e}+\beta\left(C_{m} \frac{\partial V_{m}}{\partial t}+I_{\text {ion }}-I_{\text {trans }}\right)
\end{aligned}
$$


Eqn. 4 is an elliptic equation and Eqn. 5 is a parabolic equation.

The elliptic equation is discretized by the finite element method using unstructured meshes. The result is a linear equation system of the form

$$
K_{\ell} \overline{u_{\ell}}=\bar{f}_{\ell} .
$$

This system of equation will be solved by our Parallel Toolbox.

\section{The Parallel Toolbox}

The Parallel Toolbox is a user friendly $\mathrm{C}^{++}$toolbox for the parallelization of partial differential equation solvers based on the finite element method.

In scientific computing the finite element approach is used in a variety of areas because of its flexibility towards the geometric complexity of the simulation domain. Different parallelization approaches are possible. One naturally offered by the finite element method evenly distributes the geometric elements on the processing nodes involved in the parallel computation. As a consequence some finite element nodes are on the interface between several subdomains and thus shared between several processes. We call these nodes shared nodes.

Data on subdomains can have two different representations, accumulated and distributed. Accumulated data representation means, that a process is storing the full numerical value of the nodes of its subdomain. Data stored distributed has only full value on nodes uniquely belonging to one process. On a shared node each process owning that node stores only a fraction of the full numerical value.

This definition leads to the conclusion, that accumulated and distributed data representation differ only on the shared nodes of local vectors.

A local accumulated vector $\overline{\mathfrak{u}}_{s}(s=1, \ldots, P)$ stores a part of the global vector $\overline{\mathfrak{u}}$, without changing any numerical values, and both are connected through the mapping operation (or linear map) $A_{s}$.

$$
\begin{gathered}
\overline{\mathfrak{u}}_{s}=A_{s} \overline{\mathfrak{u}} \\
A_{s}^{(i, j)}= \begin{cases}1 & \text { iff global node } \overline{\mathfrak{u}}_{j} \text { is stored locally at } \overline{\mathfrak{u}}_{s, i} \\
0 & \text { else }\end{cases} \\
\operatorname{dim} A_{s}=\# \text { local nodes } \times \text { \# global nodes }
\end{gathered}
$$

The matrix $A_{s}$ is not stored as matrix in process $s$ but as vector $l 2 g$ (local to global node numbering) with $l 2 g_{i}:=j$. This vector is used to setup the corresponding communication routines. Note that $R:=\sum_{s=1}^{P} A_{s}^{T} A_{s}$ results in a diagonal matrix containing the number of subdomains a node belongs to. Therefore, applying the local diagonal matrix $A_{s} R^{-1} A_{s}^{T}$ to a local vector results in the partition of unity.

A local distributed vector holds only a fraction of the data values in its shared nodes. To get the global data values, processes owning a certain node need to add up their values and next neighbour communication is needed. Therefore the global vector $\bar{u}$ can 
be obtained by applying the transposed mapping operation $\left(A_{s}^{T}\right)$ on the local vectors $\overline{\mathrm{u}}_{s}$ and adding up the results over all processes.

$$
\overline{\mathrm{u}}=\sum_{s=1}^{P} A_{s}^{T} \overline{\mathrm{u}}_{s}
$$

To minimize the communication between the processes, a partitioning strategy is necessary that minimizes the number of boundary nodes between the subdomains. This can be achieved using partitioning tools like METIS, which automatically creates optimal partitions based on the mesh connectivity information. For the parallelization itself, the Parallel Toolbox uses the distributed memory approach via the MPI standard.

\subsection{Linear algebra operations}

The Parallel Toolbox stores the system matrix in distributed data representation. This means that the coefficient matrix $\mathrm{K}$ is defined as:

$$
\mathrm{K}=\sum_{s=1}^{p} A_{s}^{T} \mathrm{~K}_{s}^{F E M} A_{s}
$$

Where $A_{s}$ is again the global-to-local mapping and $\mathrm{K}_{s}^{F E M}$ is the local finite-element matrix in the subdomain $\Omega_{s}$.

Depending on the actual algorithm, vectors are represented as distributed or accumulated. However, algorithms are implemented in such a way that the amount of data representation conversion, and thus MPI communication, is minimal[2, §5].

The following list shows the main linear algebra operations and their needed communication:

\section{No communication:}

- Matrix-vector multiplication (product of distributed matrix and accumulated vector; result is a distributed vector)

- Vector algebra with vectors of the same data representation

- Converting an accumulated vector into a distributed one

\section{Next neighbour communication:}

- Converting a distributed vector into an accumulated one

\section{Global (reduce) communication:}

- Computing a vector dot product

\subsection{The communicator object}

It is necessary in parallel linear algebra routines to communicate information that is located on the shared boundary nodes. To make this communication transparent for the user of the toolbox, a communicator object is created.

With the partitioning information of the elements - basically a one-to-one mapping of elements to processors - and the mesh connectivity information it is possible to derive the complete communication setup for parallel algorithms. 
In each processing node the constructor of the communicator object only needs the global indices of the local nodes. Then it calculates the local shared nodes by sending its global node numbers to every other processor and intersecting its set of global nodes, with the sets received from the other processors.

After construction, all the relevant operations in parallel linear algebra can be executed using the three communication functions offered by the communicator object:

Accumulate: Converts a distributed vector into a accumulated one, using next neighbour communication to sum up values at nodes shared with neighbouring processes.

Distribute: Converts an accumulated vector into a distributed one without communication.

Collect: Sums globally a scalar value or a vector, e.g. used in a vector dot product.

For further information about the Parallel Toolbox and its parallelization concepts we refer to [6].

\subsection{The algebraic multigrid preconditioner}

In multigrid theory one has to assume, that there exists a series of regular (finiteelement) meshes $\tau_{k}, k=1, \ldots, \ell$, where the coarser mesh $\tau_{i}$ can be derived from the finer mesh $\tau_{i+1}$. This leads to a series of systems of linear equations

$$
K_{i} \bar{u}_{i}=\bar{f}_{i}
$$

where $K_{i}$ is the stiffness matrix of the according finite-element mesh $\tau_{i}$. The main idea of multigrid methods is, that the error of the solution can be represented as the sum of the eigenfrequencies of the matrix $K$ and lower frequency parts of the error can be reduced faster on a coarser grid.

Algebraic multigrid means, that the hierarchy of meshes $\tau_{i}$ and appropriate operators $K_{i}(i<\ell)$ are created algebraically from the given finest mesh information $K_{\ell}$ (and $\left.\tau_{\ell}\right)$.

The idea of the coarsening algorithm is to part the set of all nodes $I$ in a set of coarse grid nodes $C$ and a set of fine grid nodes $F$ (see [8]).

$$
C \cap F=\varnothing, \quad I=C \cup F
$$

The matrix graph is assumed to be symmetric - i.e. originates form a symmetric matrix - and represents the connectivity information of the nodes. An off-diagonal matrix entry can be seen as a connection of one node to another node, for example if $i \neq j$ and $A_{i j} \neq 0$ then node $i$ is connected to node $j$.

A node connection is called strong if

$$
\left|A_{i j}\right|>\varepsilon\left|A_{i i}\right|
$$

otherwise it is called weak.

The algorithm starts with selecting the first node in the set $I$ and defining it as a coarse grid node. All nodes that are strongly connected to the first node are defined as 
fine grid nodes. After this initial step the next node that is yet neither fine nor coarse is selected as a coarse grid node and all nodes strongly connected to this node are set as fine grid nodes in the same way as for the first node. The algorithm terminates when all nodes are either fine or coarse grid nodes. The set of coarse grid nodes now defines a coarse grid on which another coarsening algorithm can be applied, thus creating a hierarchy of grids (and linear equation systems). Between two levels in the equation system hierarchy, also proper restriction / interpolation operators and smoothers are needed. Straightforward implementations of the Omega-Jacobi and Gauß-Seidel iterative solvers were used as smoothers. Because both smoothing algorithms need nextneighbour-communication, the AMG performance depends on a fast intercommunication network. In future versions of the Parallel Toolbox, also block-wise multigrid implementations will be available similar to the smoothers used in [3].

Although the algebraic multigrid method can be used as a solver itself, our Parallel Toolbox uses it as a preconditioner for a conjugate gradient solver. For more information about algebraic multigrid methods and their parallelization we refer to [4,2] and [11].

\subsection{The GPU implementation}

The main advantage of the GPU consists in the much higher memory bandwidth with respect to the GPU memory and in the better arithmetic performance in comparison to a CPU. The potential speedup of the GPU is at least one order of magnitude but it can only be achieved for massive parallel algorithms taking into account coalesce memory access of the data needed in the single threads. Additionally, unnecessary memory transfer between CPU memory and the limited GPU memory has to be avoided (see [6]).

The GPU algorithms use the same concepts of domain decomposition and data representation as their CPU counterparts. The main difference is, that algorithm parts that would run sequentially on each CPU process - e.g. a matrix-vector product or vector arithmetic - are now implemented in CUDA. By other words, the computationally challenging CPU routines were replaced by GPU routines of higher performance.

In the conjugate gradient solver the equation system, the initial guess and the righthand-side are transferred to the GPU memory and then all linear algebra operations needed in a conjugate gradient algorithm (matrix-vector product, vector dot product and vector arithmetic) are executed on the GPU. After that, the computed solution is transferred back to CPU memory. If absolute error tolerance is needed, then also an accumulation operation, and therefore MPI communication over the CPU memory, is required.

The algebraic multigrid coarsening is part of the setup and not implemented on GPUs. The interpolation and restriction operations during each solution step are matrixvector multiplications and can easily be executed on GPUs. For practical reasons, only the Omega-Jacobi smoother is implemented.

Every MPI process uses one GPU (CUDA device) for its computations and the user has basically the same freedoms in his choice of parallelization level and machine topology as on a CPU computation. 

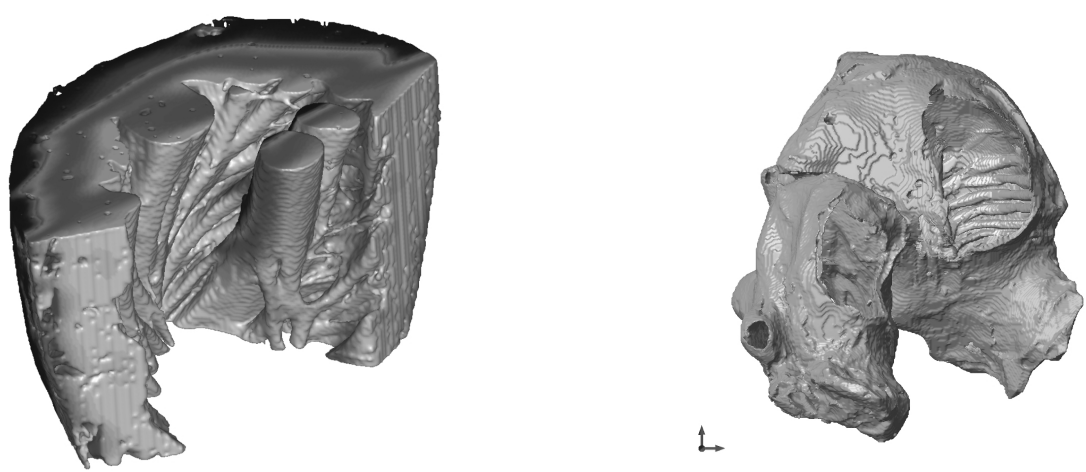

Fig. 1. The wedge_35um (left) and atria (right) geometries

\section{Benchmarks}

\subsection{Geometries}

In order to test the performance of the parallel solver we generated a stiffness matrix of an 3D finite-element unstructured mesh and solved the linear equation system $K \bar{u}=\bar{f}$. The meshes were provided by the CARP project (see [7] and [9]).

The mesh for the CPU benchmarks is called wedge_35um. It has approximately 150 million elements and 24.5 million nodes.

On the GPU clusters a geometry named atria is used. It consists of 11 million elements and 2 million nodes.

\subsection{Cluster specifications}

The CPU computations were made on the CINECA SP6 cluster. It features an IBM pSeries 575 architecture with IBM Power6 4.7 GHz processors and 5376 computing cores and a Infiniband $\mathrm{x} 4$ DDR network.

The GPU benchmarks were made on the gpuser GPU Cluster of the University of Wyoming. It consists of 7 computing nodes connected by Gigabit Ethernet and a total sum of 28 GeForce GTX 295 graphics cards. It should be noted, that the GTX 295 features two GPUs per card, so each computing node contains 4 GTX 295 cards and 8 GPUs.

\subsection{Results}

The CPU benchmarks were made on the wedge_35um geometry. The linear equation system had 24.5 million unknowns. The error tolerance in the AMG-CG solver was $10^{-6}$. Benchmarking started with 4 processes and went until 512 processes, always doubling the last number of processes in each new step. In order to have optimal caching behaviour, hyper-threading was not activated on the Power6 processors, so each MPI process used one processor core. 


\begin{tabular}{cccc}
\hline \# processes & Solving time $[\mathrm{s}]$ & iterations & \# AMG levels \\
\hline 4 & 64.51 & 19 & 6 \\
8 & 40.56 & 20 & 6 \\
16 & 14.51 & 19 & 6 \\
32 & 6.33 & 19 & 6 \\
64 & 2.9 & 20 & 6 \\
128 & 1.77 & 20 & 6 \\
$\mathbf{2 5 6}$ & $\mathbf{1 . 2 3}$ & $\mathbf{2 1}$ & $\mathbf{6}$ \\
512 & 1.42 & 21 & 6 \\
\hline
\end{tabular}

Table 1. Wall clock solving time of the CPU algorithm

Table 1 shows the CPU benchmark results. It took 4 processes about 65 seconds to compute the solution. The shortest solution time of 1.23 seconds was achieved with 256 processes. After that, the computation time was stagnating. The reasons for that will be discussed later on.

The parallel efficiency has been computed from these results using 4 processes as baseline, i.e. the efficiency for $p$ processors is calculated as $\frac{4 t_{4}}{p t_{p}}$.

An optimal linear scalability occurs, when the amount of processes doubles and the computation time halves, i.e. the efficiency is one. The efficiency in each parallelization step can be seen in figure 2 on the left. Between 4 and 256 processes the scalability is quite linear and thus the algorithm efficient. Because of caching effects on the IBM Power6 processors, also a superlinear region between 16 and 128 was noticed.

The performance drop on the highest parallelization level can be explained by analysing the amount of network communication in comparison to the local problem sizes. The amount of shared nodes of the geometry increased dramatically on high parallelization levels. If the local problems get too small, the performance gain achieved by reducing the local problem size is nihilated by the increased communication time.

The GPU benchmarks were made on a 2 million unknowns system generated from the atria geometry. The error tolerance of the AMG-CG solver was again $10^{-6}$ and between 2 and 16 processes have been used for the computations, with one MPI process for each GPU.

\begin{tabular}{cccc}
\hline \# processes & Solving time $[\mathrm{s}]$ & iterations & \# AMG levels \\
\hline 2 & 0.4 & 20 & 7 \\
4 & 0.23 & 20 & 7 \\
6 & 0.17 & 20 & 7 \\
$\mathbf{8}$ & $\mathbf{0 . 1 4}$ & $\mathbf{2 0}$ & $\mathbf{7}$ \\
12 & 1.2 & 20 & 7 \\
16 & 1.3 & 20 & 7 \\
\hline
\end{tabular}

Table 2. Wall clock solving time of the GPU algorithm 
As seen in table 2, computation times started at 0.4 seconds with 2 GPUs and reached the minimum of 0.14 seconds on 8 GPUs and on one computing node. After that, a big performance drop occurred. Using more than 8 GPUs requires a second computing node and results in a dramatic performance drop (see table 2) caused by the relatively slow network communication on the Gigabit Ethernet. This bottleneck has to be reduced technically by a faster intercommunication network.

The right part of figure 2 shows the computation efficiency. Again the performance bottleneck can be seen when more than one computing node is used.

For a direct comparison between GPU and CPU performance, the atria example was also used for a CPU benchmark. The solving time was between 9.2 seconds on 2 cores and 3.6 seconds on 16 cores. On 6 CPU cores the solution was computed in 5.8 seconds whereas on the same amount of GPUs only 0.17 seconds were needed. This resulted in a peak speedup of 34 .
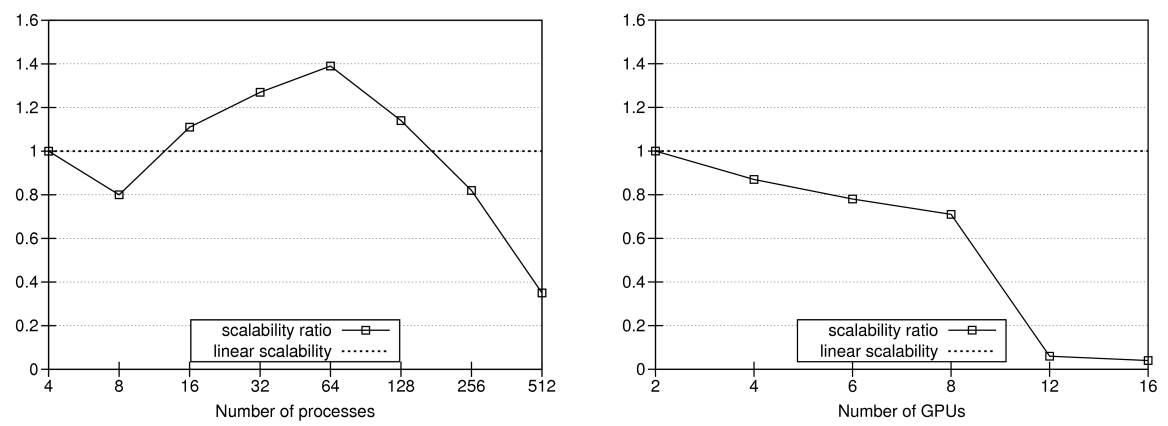

Fig. 2. Efficiency of the CPU algorithm for 24.5 mill. unknowns (left) and efficiencyof the GPU algorithm for 2 mill. unknowns (right)

\section{Conclusion}

We presented the concepts of our domain decomposition and data representation, which are the basis of the parallel linear algebra implementations. Further we investigated the algebraic multigrid method and the differences between the CPU and the GPU implementation. Finally we presented benchmarks on two geometries from the CARP project and both the CPU and GPU benchmarks showed good efficiency on most parallelization levels.

The CPU algorithm proved a very good efficiency up to 256 processes. The peak performance was on 256 processes with a solution time for a 24.5 million unknowns problem of 1.23 seconds. Only on the highest parallelization level of 512 cores the performance dropped. The reason for that was the already tiny local problem size and thus the bad ratio between computation and communication.

The GPU benchmarks showed, that data transfer is vital in GPU computations. Even though the data transfer from the GPU memory to CPU memory and from one MPI- 
host to another MPI-host are overlapping, performance dropped dramatically when the benchmark was run on more then one computing node (more then 8 MPI-processes were used). In that case the relatively low Gigabit network bandwidth proved to be the performance bottleneck. In any case it should be noted, that a dramatic increase in performance, compared to CPU computations, was shown. The fastest solution time of a 2 million unknowns system was 0.14 seconds and the peak speedup compared to a CPU computation was 34 .

GPU clusters with a very high network bandwidth and low latencies(e.g. Infiniband) or with direct GPU to GPU data transfer technology, would help to improve parallel GPU efficiency. Although the supreme computation performance of modern GPUs will probably always render data transfer speed problematic for keeping efficiency rates high.

Using block solvers and block preconditioner, where operations needing more network bandwidth are only used locally on the computing nodes and between the several nodes only operations of lesser network demands are used, could be a solution for the network limitations. This hypothesis is a subject of future research.

\section{Acknowledgment}

This work has been partially supported by Austrian Science Fund (FWF): F 3201-N18.

\section{References}

1. M. Brezina, A. J. Cleary, R. B. Falgout, V. E. Henson, J. E. Jones, T. A. Manteuffel, S. F. McCormick, and J. W. Ruge. Algebraic multigrid based on element interpolation AMGe. SIAM J. Sci. Comput., 22(5):1570-1592, 2000.

2. C. Douglas, G. Haase, and U. Langer. A Tutorial on Elliptic PDE Solvers and Their Parallelization. Software, Environments, and Tools,. SIAM, Philadelphia, 2003.

3. D. Göddeke. Fast and Accurate Finite-Element Multigrid Solvers for PDE Simulations on GPU Clusters. PhD thesis, Technische Universität Dortmund, Fakultät für Mathematik, May 2010. http://hdl.handle.net/2003/27243.

4. G. Haase, M. Kuhn, and S. Reitzinger. Parallel AMG on distributed memory computers. SIAM SISC, 24(2):410-427, 2002.

5. V. E. Henson and U. M. Yang. BoomerAMG: a Parallel Algebraic Multigrid Solver and Preconditioner. Applied Numerical Mathematics, 41:155-177, 2002.

6. M. Liebmann. Efficient PDE Solvers on Modern Hardware with Applications in Medical and Technical Sciences. PhD in natural sciences, Institute of Mathematics and Scientific Computing - Karl Franzens University Graz, 2009.

7. A. J. Prassl, F. Kickinger, H. Ahammer, V. Grau, J. E. Schneider, E. Hofer, E. J. Vigmond, N. A. Trayanova, and G. Plank. Automatically generated, anatomically accurate meshes for cardiac electrophysiology problems. IEEE Trans Biomed Eng, 56(5):1318-30, 2009.

8. J. W. Ruge and K. Stüben. Efficient solution of finite difference and finite element equations by algebraic multigrid (amg). Multigrid methods for integral and differential equations (Paddon, D.J., Holstein, H., eds.). The Institute of Mathematics and ItsApplications Conference Series, page 169-212, 1985. 
9. G. Seemann, C. Höper, F. B. Sachse, O. Dössel, A. V. Holden, and H. Zhang. Heterogeneous three-dimensional anatomical and electrophysiological model of human atria. Philos Transact A Math Phys Eng Sci, 364(1843):1465-1481, Jun 2006.

10. S. Turek, D. Göddeke, C. Becker, S. H. Buijssen, and H. Wobker. FEAST - Realisation of hardware-oriented numerics for HPC simulations with finite elements. Concurrency and Computation: Practice and Expecience, 22(6):2247-2265, May 2010. Special Issue Proceedings of ISC 2008.

11. P. S. Vassilevski. Multilevel Block Factorization Preconditioners: Matrix-based Analysis and Algorithms for Solving Finite Element Equations. Springer, New York, 1st edition, 2008.

12. E. J. Vigmond, M. Hughes, G. Plank, and L. Leon. Computational tools for modeling electrical activity in cardiac tissue. Jornal of Electrocardiology, 36:69-74, 2003. 